\title{
A Response Generator with Response-Aware Encoder for Generating Specific and Relevant Responses
}

\section{So-Eon Kim ( $\sim$ sekim0211@khu.ac.kr)}

Kyung Hee University https://orcid.org/0000-0002-6395-8246

Hyun-Je Song

Jeonbuk National University

Seong-Bae Park

Kyung Hee University https://orcid.org/0000-0002-6453-0348

\section{Research Article}

Keywords: response generator, sequence-to-sequence model, response-aware, transformer architecture, natural language processing

Posted Date: February 25th, 2022

DOI: https://doi.org/10.21203/rs.3.rs-1379873/v1

License: (a) (1) This work is licensed under a Creative Commons Attribution 4.0 International License. Read Full License 


\title{
A Response Generator with Response-Aware Encoder for Generating Specific and Relevant Responses
}

\author{
So-Eon Kim ${ }^{1}$, Hyun-Je Song ${ }^{2}$ and Seong-Bae Park ${ }^{1 *}$ \\ $1^{*}$ Department of Computer Science and Engineering, Kyung Hee University, 1732 \\ Deogyeong, Yongin, 17104, Gyeonggi, Republic of Korea. \\ ${ }^{2}$ Department of Information Technology, Jeonbuk National University, 567, Baekje, \\ Jeonju, 54896, Jeollabuk, Republic of Korea.
}

*Corresponding author(s). E-mail(s): sbpark71@khu.ac.kr;

Contributing authors: sekim0211@khu.ac.kr; hyunje.song@jbnu.ac.kr;

\begin{abstract}
The dialogue data usually consist of the pairs of a query and its response, but no previous response generators have exploited the responses explicitly in their training while a response provides significant information about the meaning of a query. Therefore, this paper proposes a sequence-to-sequence response generator with a response-aware encoder. The proposed generator exploits golden responses by reflecting them into a query representation. For this purpose, the response-aware encoder adds a relevancy scorer layer to the transformer encoder that calculates the relevancy of query tokens to a response. However, golden responses are available only during training the response generator and unavailable at the inference time. As a solution to this problem, the joint learning of a teacher and a student relevancy scorer is adopted. That is, at the training time, both the teacher and the student relevancy scorers are optimized but the decoder generates a response using only the relevancy of the teacher scorer. However, at the inference time, the decoder uses that of the student scorer. Since the student scorer is trained to minimize the difference from the teacher scorer, it can be used to compute the relevancy for a prospective response.
\end{abstract}

Keywords: response generator, sequence-to-sequence model, response-aware, transformer architecture, natural language processing

\section{Introduction}

A number of studies have shown that the generation of a response unrelated to a query is one of the most critical problems in response generation $[1,2]$, and thus have explored the reasons. The unrelated responses are categorized into ungrammatical, irrelevant, illogical, and over-general ones [3]. The generation of ungrammatical responses is now decreasing due to recent pre-trained language models, and the illogical responses are nearly inevitable in the end-to-end architecture [4]. As a result, the task of response generation focuses mainly on reducing over-general and irrelevant responses $[5,6]$.

One reason for general responses is that the traditional loss functions such as maximum likelihood have a tendency to assign a high probability to safe responses. To prevent a response generator from preferring general responses to specific ones, $\mathrm{Li}$ et al. modeled the bidirectional influence 
between a query and its response by proposing the maximum mutual information as a loss function [7], and $\mathrm{Wu}$ et al. added a max-marginal ranking regularization term to the cross-entropy loss [8]. However, since they focused only on avoiding safe responses, their methods are ineffective in reducing other types of unrelated responses.

Some recent studies have found that it helps a response generator evade the irrelevant responses to resolve the lack of background knowledge [3]. Thus, they tried to inject some external knowledge into a response generator. Ghazvininejad et al. adopted unstructured texts [9] and $\mathrm{Wu}$ et al. chose a structured graph as external knowledge for supplying the background knowledge for dialogues [10]. The search for the knowledge adequate to a query is a must in this approach, but it is not easy to search it when the query is short as in many dialogue tasks.

Both irrelevant and over-general responses are actually originated from unsuccessful capturing of the core meaning of a query. Existing response generators usually capture the core meaning only with a query and do not consider a user response even though user responses are available for their training. It is also known that a human utterer designs her utterance to induce a certain response of a hearer and the response by the hearer is made by taking the core meaning of the utterance into consideration [11]. Therefore, both a query and its prospective response are required to encode the query to generate an appropriate response to the query. However, at least to our knowledge, there is no study to model prospective responses explicitly for query representation.

This paper proposes a novel response generator that generates a relevant and specific response to a query. The proposed response generator reflects the response of a query to the query representation. For this, the encoder of the proposed generator imposes the importance weight to query tokens according to the relevancy of the tokens to a response. The key problem here is that the response is not available at the inference time. As a solution to this problem, the joint learning of teacher and student networks [12] is adopted where the input for one network (teacher) is a pair of a query and a response and that for the other network (student) is just a query. The two networks are trained to minimize the difference of their posterior distributions. Thus, the response generator uses the teacher network at the training time and the student network at the inference time to obtain a query representation. As a result, a query is encoded as a vector attended by its real response at the training time and by a prospective response at the inference time. The effectiveness of this query representation is shown by applying the response-aware encoder to a pre-trained sequenceto-sequence model such as MASS [13] and BART [14].

\section{Related Work}

\subsection{Avoiding Inappropriate Responses}

A number of efforts have been made to minimize inappropriate responses since a sequenceto-sequence model was introduced to response generation. However, $\mathrm{Wu}$ et al. found out that the standard loss for training a response generator prefers high-frequent tokens to low-frequent ones [8]. As a result, new losses for compensating for low-frequent tokens have been proposed. For instance, Jiang et al. proposed the frequency-aware cross-entropy loss [15]. However, they focused only on avoiding general responses, while inappropriate responses are categorized into four types of ungrammatical, irrelevant, illogical, and over-general responses [3].

One reason for irrelevant responses is the lack of background knowledge of a response generator. The representative approach to this problem is to provide some external information to a response generator $[16,17]$. For instance, Ghazvininejad et al. used an unstructured text as external knowledge for a fully data-driven neural dialogue system [9], while Zhou et al. and $\mathrm{Wu}$ et al. adopted a knowledge graph to provide common knowledge to a dialogue response generator $[10,18]$. Another way to provide background knowledge is to use a dialogue corpus which contains a specific type of information. Zhang et al. and Rashkin et al. exploited a corpus with persona and empathetic information respectively $[19,20]$.

\subsection{Teacher-Student Joint-Learning Framework}

Hinton et al. proposed a teacher-student framework for the first time to reduce the resource 


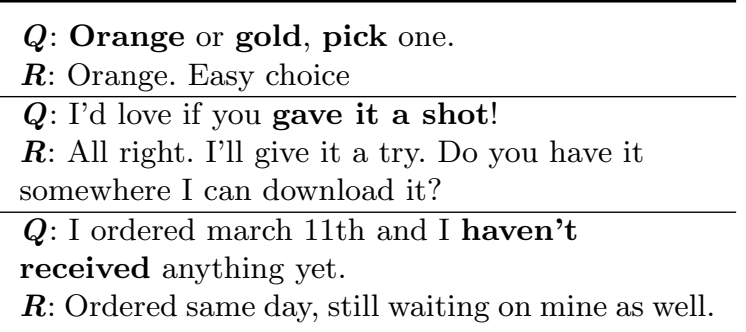

Table 1 The examples which show that the response context affects response generation in Reddit data set.

consumption of deep neural networks [21]. After that, a number of studies have been proposed to deliver knowledge of a teacher network to a student network [22], but the framework is used for a different goal in the response generation. Since a student network is trained to mimic its teacher network under this framework, Lian et al. have used the framework to allow a model to exploit the information available only when training the model [12]. Following this study, Wu et al. used a teacher network to guide a student network for knowledge selection using both a query and a response [23], and Bai et al. did it using a dialogue goal and dialogue history [24].

\subsection{Implicit Reflection of Responses}

The fact that most end-to-end response generators are trained with the pairs of a query and its response implies that they are taught implicitly to reflect the response somehow or other. MASS [13], BART [14], and T5 [25] learn the relation between a query and a response at the cross attention layer of the decoder, and DialoGPT [26] and UBAR [27] learn it at the self-attention layer. However, the effect of using the responses is limited due to their implicit reflection. Nevertheless, there is no study yet to exploit the responses explicitly to the best of our knowledge.

\section{Factors Affecting Query Representation}

The representation of a query is affected by two kinds of factors. One is relative importance among the words within a query. A query contains, in general, a few important words to represent its core meaning. Thus, many previous studies tried to find out the important words by an attention mechanism [28, 29]. However, a query by itself is insufficient to understand the core meaning of a query fully. According to the work of Grice et al., a query is designed by considering its prospective response [11]. Thus, the other factor that affects the query representation is a prospective response of the query.

Table 1 enumerates three example pairs of a query and its response extracted from Reddit data set to show the insufficiency of a query for capturing its core meaning in a single-turn dialogue. $\boldsymbol{Q}$ and $\boldsymbol{R}$ in this table indicate a query and its response, respectively. The important words of a query are marked in boldface. In the first example, it can be figured out easily that an utterer for $\boldsymbol{Q}$ expects a respondent to answer with 'orange' or 'gold'. Thus, 'orange' and 'gold' should be regarded as important words. Note that the actual response in $\boldsymbol{R}$ is made with 'orange'. In addition, the word 'choice' in $\boldsymbol{R}$ is strongly associated with 'pick' in $\boldsymbol{Q}$. Therefore, 'pick' also should be an important word.

Other examples show a similar phenomenon. For instance, $\boldsymbol{R}$ contains the expressions of 'gave it a try' and 'still waiting'. Thus, the expressions of 'gave it a shot' and 'haven't received' in $\boldsymbol{Q}$ should be considered as important ones. That is, the words in a query that allow inducing a response are important ones. Therefore, such words should be focused on when generating a query representation.

\section{Response-Aware Encoder}

The proposed response generator is basically a sequence-to-sequence model of which whole structure is explained in detail in the next section. Its main distinction from other sequence-to-sequence models is the response-aware encoder that reflects both a query and a response into a query representation. Thus, the explanation about the proposed generator begins with what the structure of the response-aware encoder looks like and how the encoder is trained.

Let $\mathcal{D}=\left\{\left(q_{i}, r_{i}\right)\right\}$ denote a single-turn dialogue data set which consists of pairs of a query $q$ and a response $r$. A query is a token sequence denoted as $q_{i}=\left\langle q_{i 1}, q_{i 2}, \cdots, q_{i n}\right\rangle$, where $n$ is a query length. A response is also a token sequence denoted as $r_{i}=\left\langle r_{i 1}, r_{i 2}, \cdots, r_{i m}\right\rangle$, where $m$ is a 
response length. A sequence of embedding vectors for a query $q_{i}, \mathbf{q}_{i}=\left\langle\mathbf{q}_{i 1}, \mathbf{q}_{i 2}, \cdots, \mathbf{q}_{i n}\right\rangle$, is obtained by leveraging a transformer encoder, since a transformer encoder have shown remarkable performances in many dialogue tasks [30]. That is, the vector $\mathbf{q}_{i}$ is computed by

$$
\mathbf{q}_{i}=\operatorname{Encoder}\left(q_{i}\right)
$$

where Encoder $(\cdot)$ is a transformer encoder.

The transformer encoder reflects only the first factor explained in the previous section with its self-attention mechanism, but does not reflect the second factor since Encoder (.) does not consider any response of a query. As a result, $\mathbf{q}_{i}$ does not represent the core meaning of a query completely. To solve this problem, the tokens in a query that corresponds with a response should be more emphasized.

Let $\mathbf{r}_{i}=\left\langle\mathbf{r}_{i 1}, \mathbf{r}_{i 2}, \cdots, \mathbf{r}_{i m}\right\rangle$ be a vector sequence for $r_{i}$, where each $\mathbf{r}_{i j}$ is encoded by the transformer encoder in Equation (1). The response $r_{i}$ can be represented as a single vector $\mathbf{r}_{i}^{e}$ by applying the average pooling to $\mathbf{r}_{i}$. That is,

$$
\mathbf{r}_{i}^{e}=\frac{1}{m} \sum_{k=1}^{m} \mathbf{r}_{i k} .
$$

Then, following the work of Cai et al. [31], the relevancy of a query tokens $q_{i j}$ to $\mathbf{r}_{i}^{e}$ is obtained by

$$
e_{i j}^{t}=\phi\left(\mathbf{q}_{i j} W_{t} \mathbf{r}_{i}^{e}\right),
$$

where $\phi$ is the $E L U$ activation function and $W_{t}$ is a learnable parameter. The relevancy of $q_{i}$ to $r_{i}$ denoted as $\mathbf{R}_{i}^{t}$ is computed by applying the soft$\max$ to $e_{i j}^{t}$ 's. That is, $\mathbf{R}_{i}^{t}=\left\langle R_{i 1}^{t}, R_{i 2}^{t}, \cdots, R_{i n}^{t}\right\rangle$, where

$$
R_{i k}^{t}=\frac{\exp \left(e_{i k}^{t}\right)}{\sum_{j=1}^{n} \exp \left(e_{i j}^{t}\right)} .
$$

Since $R_{i j}^{t}$ is a scalar value which represents an importance weight of $q_{i j}$ to $r_{i}$, the final responseaware representation of $q_{i}$ is obtained by multiplying every $R_{i j}^{t}$ to its corresponding $\mathbf{q}_{i j}$. That is, the final query representation $\hat{\mathbf{q}}_{i}$ is

$$
\hat{\mathbf{q}}_{i}^{t}=\left\langle\hat{\mathbf{q}}_{i 1}^{t}, \hat{\mathbf{q}}_{i 2}^{t}, \cdots, \hat{\mathbf{q}}_{i n}^{t}\right\rangle,
$$

where $\hat{\mathbf{q}}_{i j}^{t}=R_{i j}^{t} \cdot \mathbf{q}_{i j}$.

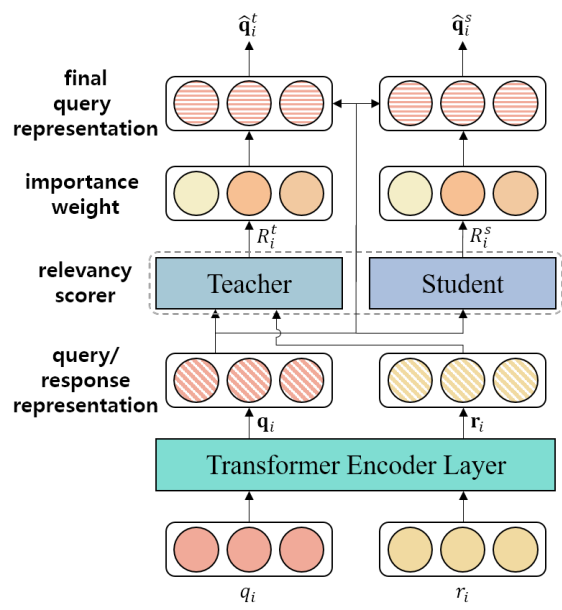

Fig. 1 The structure of the proposed response-aware encoder.

Note that $r_{i}$ 's are available only at the training time. There is no response of a query at the inference time. Therefore, it is impossible to calculate the relevancy $\mathbf{R}^{t}$ at the inference time. Inspired by the work of $\mathrm{Wu}$ et al. [23], a teacherstudent framework is adopted for distilling the knowledge of scoring $\mathbf{R}^{t}$ to a student relevancy scorer. Let the module for scoring $\mathbf{R}^{t}$ be a teacher relevancy scorer. Then, a student relevancy scorer also measures the relevancy of each query token under the circumstance in which only queries are available without their responses. For each query token vector $\mathbf{q}_{i j}$, the student scorer determines the relevancy of $q_{i j}$ by

$$
e_{i j}^{s}=\phi\left(\mathbf{q}_{i j} W_{s}\right),
$$

where $W_{s}$ is a learnable parameter. The relevancy score $\mathbf{R}_{i}^{s}=\left\langle R_{i 1}^{s}, R_{i 2}^{s}, \cdots, R_{i n}^{s}\right\rangle$ is then obtained by applying the softmax function to $e_{i j}^{s}$ 's. That is,

$$
R_{i k}^{s}=\frac{\exp \left(e_{i k}^{s}\right)}{\sum_{j=1}^{n} \exp \left(e_{i j}^{s}\right)} .
$$

Whereas $\mathbf{R}_{i}^{s}$ is the relevancy of $q_{i}$ to $r_{i}$, it is trained only with the queries which leads to a large discrepancy from $\mathbf{R}_{i}^{t}$. To make the discrepancy minimized, the student relevancy scorer is trained with the Kullback-Leibler loss

$$
\mathcal{L}_{k d}=K L D\left(\mathbf{R}_{i}^{t}, \mathbf{R}_{i}^{s}\right) .
$$



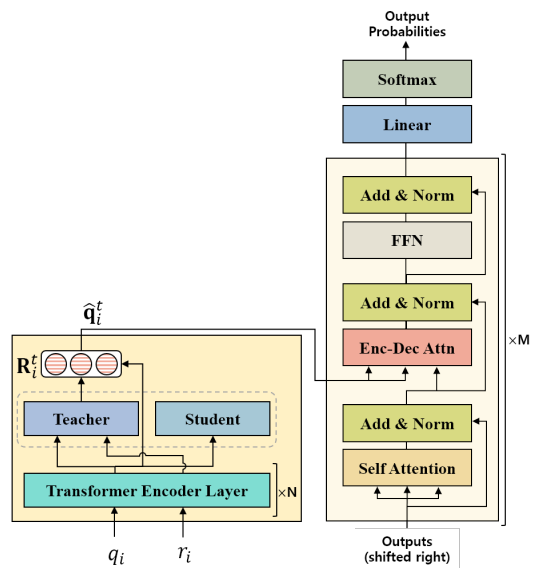

(a) Training - Relevancy Score at Encoding

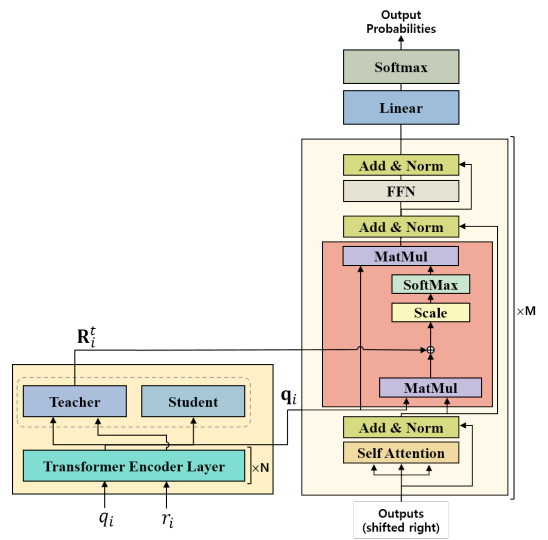

(c) Training - Relevancy Score at Decoding

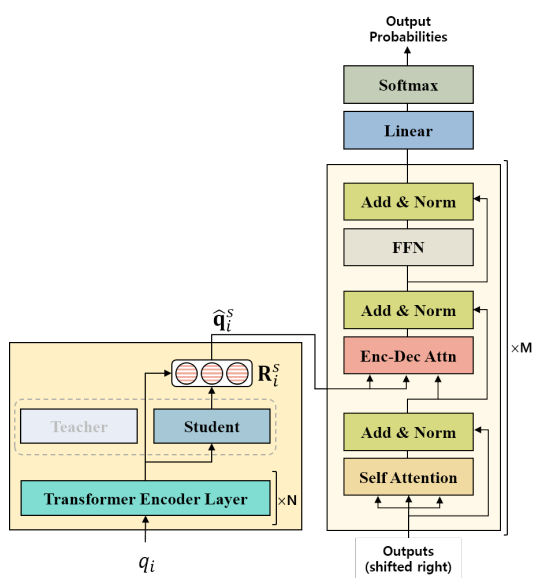

(b) Inference - Relevancy Score at Encoding

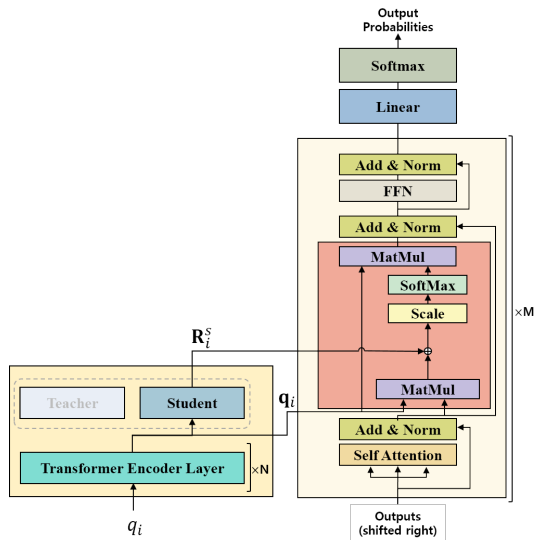

(d) Inference - Relevancy Score at Decoding

Fig. 2 The overall architecture of the proposed response generator. (a) and (b) use $\mathbf{R}_{i}^{t}$ and $\mathbf{R}_{i}^{s}$ for the encoder respectively, while (c) and (d) use them as an attention for the decoder.

The smaller $\mathcal{L}_{k d}$ is, the more similar $\mathbf{R}_{i}^{s}$ becomes to $\mathbf{R}_{i}^{t}$. As a result, if the student relevancy scorer is trained to minimize the loss in Equation (7), then it also gets able to compute the similarity of $q_{i}$ to $r_{i}$. Then, the final query representation $\hat{\mathbf{q}}_{i}$ by the student relevancy scorer is

$$
\hat{\mathbf{q}}_{i}^{s}=\left\langle\hat{\mathbf{q}}_{i 1}^{s}, \hat{\mathbf{q}}_{i 2}^{s}, \cdots, \hat{\mathbf{q}}_{i n}^{s}\right\rangle,
$$

where $\hat{\mathbf{q}}_{i j}^{s}=R_{i j}^{s} \cdot \mathbf{q}_{i j}$.

The structure of the proposed response-aware encoder is shown in Figure 1. The encoder consists of two modules: a transformer encoder layer and relevancy scorers. The transformer encoder layer transforms a query $q_{i}$ and its response $r_{i}$ into vector representations of $\mathbf{q}_{i}$ and $\mathbf{r}_{i}$. After that, the relevancy of $\mathbf{q}_{i}$ to $\mathbf{r}_{i}$ is applied to $\mathbf{q}_{i}$, where the relevancy is computed by two scorers. The teacher scorer computes the relevancy $\mathbf{R}_{i}^{t}$ with Equation (2) and (3) using both $\mathbf{q}_{i}$ and $\mathbf{r}_{i}$, while the student scorer computes the relevancy $\mathbf{R}_{i}^{s}$ with Equation (5) and (6) using only $\mathbf{q}_{i}$. Then, the final query representation for $q_{i}$ is obtained by both scorers. The teacher scorer represents $q_{i}$ as $\hat{\mathbf{q}}_{i}^{t}$ in Equation (4) and the student scorer does as $\hat{\mathbf{q}}_{i}^{s}$ in Equation (8). However, note that both the teacher scorer and the student scorer are trained at the training time, but only the student scorer is used to represent $q_{i}$ at the inference time.

\section{Response Generation by Response-Aware Encoder}

Figure 2 shows the overall structure of the proposed response generator which utilizes the response-aware encoder. The response generator 
has an encoder-decoder structure. Thus, it is based on the pre-trained sequence-to-sequence models that are BART [14] and MASS [13]. To avoid excessive resource consumption and efficient learning, the parameters of the transformer encoder layer in the response-aware encoder and the decoder are all initialized with those of BART or MASS. Therefore, if the proposed generator is initialized by BART, it is called as Response-Aware BART (RA-BART). Similarly, it is ResponseAware MASS (RA-MASS) if it is initialized by MASS.

There could be two models to utilize $\mathbf{R}_{i}^{t}$ and $\mathbf{R}_{i}^{s}$. One is to make the encoder use them as explained in the previous section. Figure 2-(a) and (b) correspond to this model. In this model, the transformer decoder receives a query representation by the response-aware encoder at the encoder-decoder attention layer as a context vector. Since a response $r_{i}$ for every query $q_{i}$ is available at the training time, $\hat{\mathbf{q}}_{i}^{t}$ from the teacher relevancy scorer is used as an input for the decoder (see Figure 2-(a)). However, at the inference time, there is no response for $q_{i}$. Thus, $\hat{\mathbf{q}}_{i}^{s}$ from the student relevancy scorer is used at the inference time as shown in Figure 2-(b).

The other model to utilize $\mathbf{R}_{i}^{t}$ and $\mathbf{R}_{i}^{s}$ is to use them at the decoder, and Figure 2-(c) and (d) show this model. This model regards $\mathbf{R}_{i}^{t}$ and $\mathbf{R}_{i}^{s}$ as an additional attention for the encoder output that comes from a response. Let $Q, K$, and $V$ are a query, a key and a value matrix for the multi-head attention respectively and $\mathbf{r}$ be a relevancy score vector. Then, the scaled-dot product attention at the encoder-decoder attention layer is defined as

$$
\operatorname{Attention}(Q, K, V, \mathbf{r})=\operatorname{softmax}\left(\frac{Q K^{T} \circ \mathbf{r}}{\sqrt{d_{k}}} V\right),
$$

where $d_{k}$ is the embedding dimension and $\circ$ is the Hadamard-product between a matrix and a vector. The vector $\mathbf{r}$ in this equation is $\mathbf{R}_{i}^{t}$ at the training time and $\mathbf{R}_{i}^{s}$ at the inference time.

The label smoothed cross-entropy loss $\mathcal{L}_{c e}$ [32] is used for training the response generator. Thus, the loss for training the whole response generator is a combination of $\mathcal{L}_{k d}$ in Equation (7) and $\mathcal{L}_{c e}$. $\mathcal{L}_{k d}$ amis at forcing the student relevancy scorer to distill the knowledge of the teacher relevancy scorer and $\mathcal{L}_{c e}$ compels the response generator to generate an answer similar to a golden response.
Table 2 Simple statistics on the data sets.

\begin{tabular}{l|c|c}
\hline & Reddit & Persona-Chat \\
\hline No. of training pairs & $1,352,961$ & 108,217 \\
No. of validation pairs & 40,000 & 13,445 \\
No. of test pairs & 40,000 & 14,520 \\
\hline
\end{tabular}

That is, the whole loss for the proposed response generator is

$$
\mathcal{L}=\mathcal{L}_{c e}+\alpha \mathcal{L}_{k d}
$$

where $\alpha$ is a hyper-parameter.

\section{Experiments}

\subsection{Data Sets}

Two benchmark data sets are used to verify the performance of the proposed response generator. One is the Reddit data set [18] and the other is the Persona-Chat data set [19]. Both data sets are composed of open-domain dialogues. Each dialogue of the Reddit data set is a single-turn query-response pair, but the Persona-Chat data set consists of multi-turn dialogues. The queries and responses shorter than four words or longer than 30 words were excluded from the Reddit data following the study of $\mathrm{Wu}$ et al. [10]. After this pre-processing, the Reddit data set contains $1,352,961$ training pairs, 40,000 validation pairs, and 40,000 test pairs. The multi-turn dialogues of the Persona-chat data set are converted to singleturn ones as done in the study of Feng et al. [33]. After the conversion, the data set contains 108,217 training pairs, 13,445 validation pairs, and 14,520 test pairs. Table 2 summarizes the statistics on the data sets used for the experiments below.

Table 3 Comparisons about how to utilize $\mathbf{R}_{i}^{s}$ 's.

\begin{tabular}{c|ccc}
\hline Methods & Emb avg & BLEU-1 & Entropy \\
\hline MASS - Encoder & 0.862 & 19.85 & 10.18 \\
MASS - Attention & 0.861 & 19.28 & 10.01 \\
\hline BART - Encoder & 0.859 & 19.47 & 10.16 \\
BART - Attention & 0.857 & 19.13 & 10.11 \\
\hline
\end{tabular}

\subsection{Implementation Details}

The hyper-parameters for the transformer encoder layer and the decoder are equivalent to those of MASS or BART. RA-MASS follows the settings 
for MASS-base-uncased ${ }^{1}$ and RA-BART obeys those for bart.base ${ }^{2}$. RA-MASS and RA-BART share many parameter values. For instance, the dimension of embedding vectors is 768 , and that of the inner-layers of feed-forward networks is 3,072 . The number of heads in multi-head attention is twelve and the number of transformer layers in the encoder and the decoder is six. The batch size of training and validation sets is 32 and the learning rate is 0.0001 . The Adam optimizer [34] is adopted with $\beta_{1}=0.9$ and $\beta_{2}=0.98$. In addition, the label smoothing of $\epsilon_{l s}=0.1$ is used with cross-entropy loss. $\alpha$ in Equation (9) is set to 0.4. RA-MASS uses an attention dropout of 0.1 and an activation dropout of 0.1 , but RA-BART does not.

\subsection{Evaluation Metrics}

The performance of a response generator is measured in four aspects: embedding, overlap, diversity and informativeness. Embedding is measured with $\mathrm{Emb}_{\text {avg }}$ and $\mathrm{Emb}_{e x}$, Overlap is with BLEU-1 and BLEU-2, Diversity is with Dist-1 and Dist-2, and Informative is assessed with Entropy. Embavg [35] is the similarity between the average of the all token embedding vectors of a golden response and that of the generated response. On the other hand, $\mathrm{Emb}_{\text {ex }}$ measures the similarity between embedding vectors using vector extrema. BLEU-1 and BLEU-2 are the ratio of uni-gram and bi-gram overlaps respectively [36], and Dist-1 and Dist-2 are the ratio of distinct uni-grams and bi-grams in all generated responses [7]. Entropy is the average word-level entropy [37] which indicates how informative a generated response is.

\subsection{Baselines}

The performance of the proposed model is compared with the following baselines:

- AS2S: a standard Seq2Seq model of RNN with an attention mechanism [28],

- COPY: a Seq2Seq based model with the copy mechanism [38],

- ConKADI: the commonsense knowledgeaware response generator [10] which shows the

\footnotetext{
${ }^{1}$ https://github.com/microsoft/MASS/tree/master/ MASS-summarization

${ }^{2}$ https://github.com/pytorch/fairseq/blob/master/ examples/bart
}

state-of-the-art performance for the Reddit data set,

- MRBD: a training framework with multi-view feature representation and bidirectional distillation [33] which shows the state-of-the-art performance for the Persona-Chat data set,

- MASS: a pre-trained transformer Seq2Seq model trained with a masked language objective [13],

- BART: a pre-trained transformer Seq2Seq model trained with a denoising objective [14].

\subsection{Experimental Results}

\subsubsection{Utilization of Relevancy Score}

There are two ways of utilizing $\mathbf{R}_{i}^{s}$ 's. One is to use them at the encoder and the other is to use them as attention for the decoder. Table 3 compares the ways empirically for the Reddit data set. Encoder in this table implies the utilization of $\mathbf{R}_{i}^{s}$ 's at the encoder while Attention indicates their utilization at the decoder. For both pretrained models of MASS and BART, Encoder outperforms Attention for all metrics. Especially, Encoder achieves much higher BLEU-1 than its corresponding Attention. To sum up, Table 3 proves that it is more efficient to apply the relevancy scores directly to the query representation than to use them indirectly through encoderdecoder attention. Therefore, $\mathbf{R}_{i}^{s}$ 's are used by the encoder at all experiments below.

\subsubsection{Comparative Study with Baselines}

Table 4 reports the experimental results about the performance of the proposed response generator. The experimental results show that the proposed generator represented as RA-MASS and RA-BART outperforms the current state-of-theart models as well as the baselines. The best model for both data sets is the proposed RAMASS. It shows the highest performance for all metrics except Dist-1 and Dist-2 for the Reddit data set. Higher embedding and BLEU imply that a model generates more relevant responses and higher entropy means that a model produces more specific responses. Thus, RA-MASS is a model that generates the most relevant and specific responses. The model with the highest diversity for the Reddit data set is ConKADI. 
ware Encoder for Generating Specific and Relevant Responses

Table 4 The empirical comparison of the proposed response generator with its baselines.

\begin{tabular}{c|cc|cc|cc|c}
\hline \multirow{2}{*}{ Metric } & Embedding & \multicolumn{2}{c|}{ Overlap (\%) } & Diversity (\%) & Informative \\
\cline { 2 - 8 } & Emb $_{\text {avg }}$ & Emb $_{\text {ex }}$ & BLEU-1 & BLEU-2 & Dist-1 & Dist-2 & Entropy \\
\hline \multicolumn{7}{c}{ Reddit data set } \\
\hline AS2S & 0.843 & 0.831 & 10.24 & 4.50 & 0.87 & 3.44 & 5.99 \\
COPY & 0.834 & 0.801 & 13.65 & 6.36 & 1.15 & 4.36 & 7.13 \\
ConKADI & 0.855 & 0.845 & - & 3.53 & $\mathbf{2 . 7 7}$ & $\mathbf{1 8 . 7 8}$ & 8.68 \\
MASS & 0.858 & 0.843 & 18.83 & 8.93 & 1.19 & 9.73 & 9.98 \\
BART & 0.853 & 0.842 & 18.16 & 8.57 & 1.07 & 9.14 & 10.08 \\
\hline RA-MASS & $\mathbf{0 . 8 6 2}$ & $\mathbf{0 . 8 5 0}$ & $\mathbf{1 9 . 8 5}$ & $\mathbf{9 . 5 1}$ & 1.32 & 10.96 & $\mathbf{1 0 . 1 8}$ \\
RA-BART & 0.859 & 0.848 & 19.47 & 9.21 & 1.17 & 9.44 & 10.16 \\
\hline \multicolumn{7}{|c|}{ Persona-Chat data set } \\
\hline AS2S & 0.812 & 0.797 & 9.59 & 3.02 & 0.68 & 2.95 & 5.40 \\
COPY & 0.822 & 0.803 & 12.85 & 5.83 & 1.02 & 5.30 & 6.84 \\
MRBD & - & - & 17.52 & - & 1.75 & 12.39 & 7.42 \\
MASS & 0.849 & 0.849 & 17.43 & 8.45 & 1.68 & 11.51 & 7.29 \\
BART & 0.843 & 0.847 & 16.95 & 8.25 & 1.48 & 10.51 & 7.18 \\
\hline RA-MASS & $\mathbf{0 . 8 5 5}$ & $\mathbf{0 . 8 5 1}$ & $\mathbf{1 8 . 1 2}$ & $\mathbf{9 . 3 4}$ & $\mathbf{1 . 9 6}$ & $\mathbf{1 2 . 9 4}$ & $\mathbf{8 . 3 1}$ \\
RA-BART & 0.852 & 0.849 & 17.86 & 9.18 & 1.86 & 12.56 & 8.12 \\
\hline
\end{tabular}

Since ConKADI exploits external knowledge to achieve high diversity, it could obtain higher diversity than RA-MASS. However, it shows lower performance for all other metrics. The state-ofthe-art model for the Persona-Chat data set is MRBD, but its performance is worse than that of RA-MASS for all metrics.

The architectures of MASS and BART are identical, while their training methods are different from each other. Since BART is trained with more diverse denoising objectives than MASS, it achieves higher performances than MASS in many tasks. However, MASS shows higher performance than BART in this task for both data sets. The lower performance of BART seems to be originated from the characteristics of dialogue data such as word omission and wide use of demonstrative pronouns. In addition, single-turn dialogues are usually short. As a result, some denoising objectives of BART act rather as an obstacle of performance degradation. This lower performance of BART yields the lower performance of RA-BART than RA-MASS.

\subsection{Case Study}

Table 5 shows two examples of how various response generators respond to a query. The queries in this figure are selected from the Reddit data set. The graph in each example depicts
Table 5 Example responses generated by various models.

$$
\text { Case Study \#1 }
$$

Query: no, but i would understand if someone would be annoyed if i had a barbecue on their father's grave. Reference Response: my dad would approve.

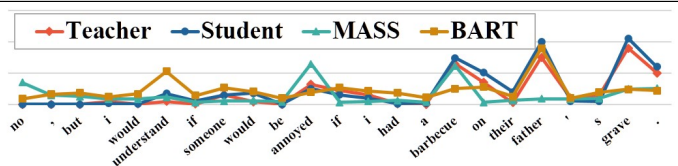

MASS: i don't think you understand how bbq works. BART: i don't know why you're being downvoted, but it's a good idea.

RA-MASS: i'd be pissed if they had a bbq on my dad's grave.

RA-BART: i don't understand why people would be annoyed by a bbq on their father's grave. Case Study \#2

Query: public markets are not the only way to sell stock.

Reference Response: yeah, but it's the best way to get a fair price.

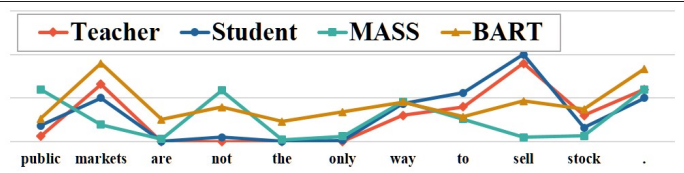

MASS: i don't think you understand how public works.

BART: they aren't, they're just not the same thing.

RA-MASS: you're right, but it's still a good way to sell stock.

RA-BART: no, they're the only way to sell stock.

the importance weights of query tokens by the 


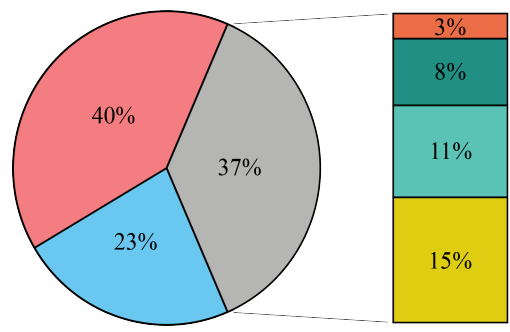

(a) MASS

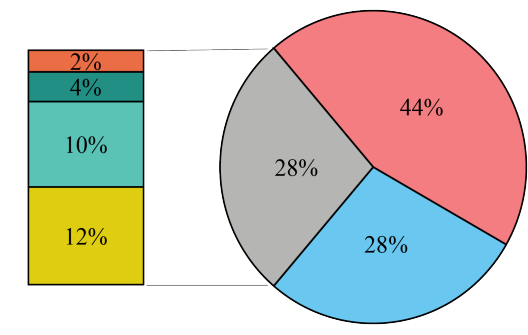

(b) RA-MASS

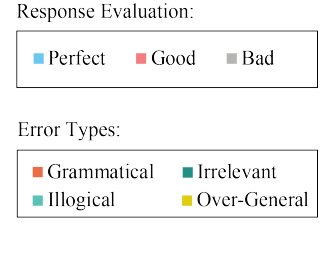

Fig. 3 Error analysis with two hundred sample responses. teacher relevancy scorer (red), the student relevancy scorer (blue), self-attention of vanilla MASS (cyan), and self-attention of vanilla BART (brown). Both relevancy scorers assign similar weights to query tokens, which implies that the student scorer trained with Equation (7) mimics its teacher scorer quite well. Therefore, it is safe to use only the student scorer at the inference time.

According to this figure, MASS and BART fail in understanding the core meaning of queries. In the first query, the teacher relevancy scorer regards "annoyed", "barbecue on", "father", and "grave" as the expressions for a core meaning. However, MASS focuses on the self-attentive word "barbecue" but does not pay any attention to "grave". As a result, it generates an inappropriate response. BART generates a very general response due to its strange self-attention. On the other hand, RA-MASS and RA-BART generate a relevant and specific response to the query due to the student scorer. That is, they generate their responses using the expressions stressed by the student scorer. As a result, the response by RAMASS contains the expressions of "pissed" and "bbq on my dad's grave", and that by RA-BART includes an expression of " $b b q$ on their father's grave".

Similarly, in the second query, the graph indicates that the key expressions are "markets" and "way to sell stock". However, MASS focuses on "public" and generates an inappropriate response. BART does not capture the meaning of the query at all and also generates an inappropriate response. On the other hand, RA-MASS and RABART focus more on the expressions with high weights by the student scorer. As a result, they produce the expression of "way to sell stock" in their responses.

\subsection{Error Analysis}

The errors by RA-MASS are analyzed for a better understanding of the limitations of the proposed response generator. For this, two hundred responses by MASS and RA-MASS are randomly selected from the test set of Reddit data. Two annotators are recruited to assign one of three labels (perfect, good, and bad) independently to every generated response following the study of Wang et al. [4]. The agreement between the annotators measured with kappa is 0.68 , which implies that the annotators made a substantial agreement. The bad responses are further categorized into ungrammatical, irrelevant, illogical, or over-general.

Figure 3 compares the responses by MASS and RA-MASS. Overall, the ratio of bad responses by RA-MASS is reduced to $28 \%$ from $37 \%$, the ratio of bad responses by MASS. As a result, the ratio of perfect responses is increased in RA-MASS, which proves that RA-MASS produces better responses. If investigating the bad responses, the ungrammatical errors take the least portion in both MASS and RA-MASS due to the generation power of the pre-trained sequence-to-sequence model. On the other hand, RA-MASS shows totally different proportions for irrelevant and over-general errors from MASS. The irrelevant responses of RAMASS take only $4 \%$, while MASS produces $8 \%$ irrelevant responses. Similarly, the ratio of overgeneral responses in RA-MASS is reduced from $15 \%$ of MASS to $12 \%$. These results prove that the proposed generator is effective in generating relevant and specific responses.

\section{Conclusions}

This paper has proposed a novel response generator that reflects a prospective response explicitly 
into query representation through a responseaware encoder. The contemplation of a response allows an encoder to capture the core meaning of a query better. As a result, the decoder could generate a more relevant and specific response. The key problem of this model is that there is no response available at the inference time. Thus, a teacher-student framework was employed which forces the student relevancy scorer to output the same relevancy scores of query tokens with the teacher relevancy scorer, where the teacher scorer is trained with the pairs of a query and a response and the student scorer is taught with only queries. The experimental results on two benchmark data sets showed that it is effective to use a prospective response of a query in encoding a query. In particular, RA-MASS outperforms all baselines for various metrics, which proves that the proposed generator produces more relevant and specific responses.

\section{Declarations}

\section{Funding}

This work was supported by the National Basic Science Research Program through the National Research Foundation of Korea(NRF) funded by the Ministry of Education (No. 2020R1A4A1018607) and Institute of Information \& Communications Technology Planning \& Evaluation (IITP) grant funded by the Korea government (MSIT) (No. 2013-0-00109, WiseKB: Big data based self-evolving knowledge base and reasoning platform).

\section{Conflict of interest/Competing interests}

The authors have no relevant financial or nonfinancial interests to disclose.

\section{Authors' contributions}

All authors contributed to the study conception and design. Material preparation, data collection were performed by So-Eon Kim and data analysis was performed by Hyun-je Song. The first draft of the manuscript was written by So-Eon Kim and reviewing and editing the manuscript were performed by Seong-bae Park. All authors read and approved the final manuscript.

\section{Availability of data and materials}

The datasets generated during and/or analysed during the current study are available in the ACL2020-ConKADI repository, https://github. com/pku-sixing/ACL2020-ConKADI.

\section{References}

[1] Serban IV, Sordoni A, Lowe R, Charlin L, Pineau J, Courville A, et al. A Hierarchical Latent Variable Encoder-Decoder Model for Generating Dialogues. In: Proceedings of the 31st AAAI Conference on Artificial Intelligence; 2017. p. 3295-3301.

[2] Huang M, Zhu X, Gao J. Challenges in Building Intelligent Open-Domain Dialog Systems. ACM Transactions on Information Systems. 2020;38(3):1-32.

[3] Wu S, Li Y, Zhang D, Wu Z. Improving Knowledge-Aware Dialogue Response Generation by Using Human-Written Prototype Dialogues. In: Proceedings of the 2020 Conference on Empirical Methods in Natural Language Processing; 2020. p. 1402-1411.

[4] Wang J, Liu J, Bi W, Liu X, He K, Xu R, et al. Improving Knowledge-aware Dialogue Generation via Knowledge Base Question Answering. In: Proceedings of the 34th AAAI Conference on Artificial Intelligence; 2020. p. 9169-9176.

[5] Ling Y, Cai F, Hu X, Liu J, Chen W, Chen H. Context-Controlled Topic-Aware Neural Response Generation for Open-Domain Dialog Systems. Information Processing \& Management. 2021;58(1):102392-102406.

[6] Sun B, Feng S, Li Y, Liu J, Li K. Generating Relevant and Coherent Dialogue Responses using Self-Separated Conditional Variational AutoEncoders. In: Proceedings of the 59th Annual Meeting of the Association for Computational Linguistics and the 11th International Joint Conference on Natural Language Processing; 2021. p. 5624-5637. 
[7] Li J, Galley M, Brockett C, Gao J, Dolan B. A Diversity-Promoting Objective Function for Neural Conversation Models. In: Proceedings of the 2016 Conference of the North American Chapter of the Association for Computational Linguistics; 2016. p. 110119.

[8] Wu B, Jiang N, Gao Z, Li M, Wang Z, Li $\mathrm{S}$, et al. Why Do Neural Response Generation Models Prefer Universal Replies? arXiv preprint arXiv:180809187. 2018;.

[9] Ghazvininejad M, Brockett C, Chang M, Dolan B, Gao J, tau Yih W, et al. A Knowledge-Grounded Neural Conversation Model. In: Proceedings of the 32nd AAAI Conference on Artificial Intelligence; 2018. p. $1-8$.

[10] Wu S, Li Y, Zhang D, Zhou Y, Wu Z. Diveirse and Informative Dialogue Generation with Context-Specific Commonsense Knowledge Awareness. In: Proceedings of the 58th Annual Meeting of the Association for Computational Linguistics; 2020. p. $5811-5820$.

[11] Grice P. Utterer's Meaning and Intentions. The Philosophical Review. 1969;78(2):147177.

[12] Lian R, Xie M, Wang F, Peng J, Wu H. Learning to Select Knowledge for Response Generation in Dialog Systems. In: Proceedings of the 28th International Joint Conference on Artificial Intelligence; 2019. p. $5081-5087$.

[13] Song K, Tan X, Qin T, Lu J, Liu TY. MASS: Masked Sequence to Sequence Pretraining for Language Generation. In: Proceedings of the 36th International Conference on Machine Learning; 2019. p. 5926-5936.

[14] Lewis M, Liu Y, Goyal N, Ghazvininejad M, Mohamed A, Levy O, et al. Bart: Denoising Sequence-to-sequence Pre-training for Natural Language Generation, Translation, and Comprehension. arXiv preprint arXiv:191013461. 2019;.
[15] Jiang S, Ren P, Monz C, Rijke M. Improving Neural Response Diversity with FrequencyAware Cross-Entropy Loss. In: Proceddings of the Web Conference 2019; 2019. p. 28792885 .

[16] Young T, Cambria E, Chaturvedi I, Zhou H, Biswas S, Huang M. Augmenting End-toEnd Dialogue Systems With Commonsense Knowledge. In: Proceedings of the 32nd AAAI Conference on Artificial Intelligence; 2018. p. $4970-4977$.

[17] Liu Z, Niu ZY, Wu H, Wang H. Knowledge Aware Conversation Generation with Explainable Reasoning over Augmented Graphs. In: Proceedings of the 2019 Conference on Empirical Methods in Natural Language Processing and the 9th International Joint Conference on Natural Language Processing; 2019. p. 1782-1792.

[18] Zhou H, Young T, Huang M, Zhao H, Xu J, Zhu X. Commonsense Knowledge Aware Conversation Generation with Graph Attention. In: Proceedings of the 27th International Joint Conference on Artificial Intelligence; 2018. p. 4623-4629.

[19] Zhang S, Dinan E, Urbanek J, Szlam A, Kiela D, Weston J. Personalizing Dialogue Agents: I Have a Dog, Do You Have Pets Too? In: Proceedings of the 56th Annual Meeting of the Association for Computational Linguistics; 2018. p. 2204-2213.

[20] Rashkin H, Smith E, Li M, Boureau YL. Towards Empathetic Open-domain Conversation Models: A New Benchmark and Dataset. In: Proceedings of the 57th Annual Meeting of the Association for Computational Linguistics; 2019. p. 5370-5381.

[21] Hinton G, Vinyals O, Dean J. Distilling the Knowledge in a Neural Network. In: Proceedings of NIPS 2014 Workshop on Deep Learning and Representation Learning; 2014. p. $1-9$.

[22] Menon A, Rawat A, Reddi S, Kim S, Kumar S. A statistical perspective on distillation. In: Proceedings of the 38th International 
Conference on Machine Learning; 2021. p. 7632-7642.

[23] Wu S, Li Y, Zhang D, Zhou Y, Wu Z. TopicKA: Generating Commonsense KnowledgeAware Dialogue Responses Towards the Recommended Topic Fact. In: Proceedings of the 29th International Joint Conference on Artificial Intelligence; 2020. p. 37663772. Available from: https://doi.org/10. 24963/ijcai.2020/521.

[24] Bai J, Yang Z, Liang X, Wang W, Li Z. Learning to Copy Coherent Knowledge for Response Generation. In: Proceedings of the 35th AAAI Conference on Artificial Intelligence; 2021. p. 12812-12820.

[25] Raffel C, Shazeer N, Roberts A, Lee K, Narang S, Matena $M$, et al. Exploring the Limits of Transfer Learning with a Unified Text-to-Text Transformer. Journal of Machine Learning Research. 2020;21:1-67.

[26] Zhang Y, Sun S, Galley M, Chen YC, Brockett C, Gao X, et al. DIALOGPT : Large-Scale Generative Pre-training for Conversational Response Generation. In: Proceedings of the 58th Annual Meeting of the Association for Computational Linguistics; 2020. p. 270-278.

[27] Yang Y, Li Y, Quan X. UBAR: Towards Fully End-to-End Task-Oriented Dialog System with GPT-2. In: Proceedings of the 35th AAAI Conference on Artificial Intelligence; 2021. p. $14230-14238$.

[28] Bahdanau D, Cho K, Bengio Y. Neural Machine Translation by Jointly Learning to Align and Translate. In: Proceedings of 3rd International Conference on Learning Representations; 2015. p. 1-15.

[29] Shan C, Zhang J, Wang Y, Xie L. Attention-based End-to-End Models for Small-Footprint Keyword Spotting. In: Proceedings of the Interspeech 2018; 2018. p. 2037-2041.

[30] Oluwatobi O, Mueller E. Dlgnet: A Transformer-Based Model for Dialogue Response Generation. In: Proceedings of the 2nd Workshop on Natural Language Processing for Conversational AI; 2020. p. $54-62$.

[31] Cai D, Wang Y, Bi W, Tu Z, Liu X, Shi S. Retrieval-guided Dialogue Response Generation via a Matching-to-Generation Framework. In: Proceedings of the 2019 Conference on Empirical Methods in Natural Language Processing and the 9th International Joint Conference on Natural Language Processing; 2019. p. $1866-1875$.

[32] Szegedy C, Vanhoucke V, Ioffe S, Shlens J, Wojna Z. Rethinking the Inception Architecture for Computer Vision. In: Proceedings of the IEEE Conference on Computer Vision and Pattern Recognition; 2016. p. 2818-2826.

[33] Feng S, Ren X, Li K, Sun X. Multi-View Feature Representation for Dialogue Generation with Bidirectional Distillation. In: Proceedings of the 35th AAAI Conference on Artificial Intelligence; 2021. p. 12812-12820.

[34] Kingma D, Ba J. Adam: A Method for Stochastic Optimization. In: Proceedings of International Conference on Learning Representations; 2015. p. 1-15.

[35] Liu CW, Lowe R, Serban IV, Noseworthy M, Charlin L, Pineau J. How NOT To Evaluate Your Dialogue System: An Empirical Study of Unsupervised Evaluation Metrics for Dialogue Response Generation. In: Proceedings of the 2016 Conference on Empirical Methods in Natural Language Processing; 2016. p. $2122-2132$.

[36] Li J, Monroe W, Shi T, Jean S, Ritter A, Jurafsky D. Adversarial Learning for Neural Dialogue Generation. In: Proceedings of the 2017 Conference on Empirical Methods in Natural Language Processing; 2017. p. 2157-2169.

[37] Mou L, Song Y, Yan R, Li G, Zhang L, Jin Z. Sequence to Backward and Forward Sequences: A Content-Introducing Approach to Generative Short-Text Conversation. In: Proceedings of the 26th International Conference on Computational Linguistics; 2016. p. 
3349-3358.

[38] Zhu W, Mo K, Zhang Y, Zhu Z, Peng X, Yang Q. Flexible End-to-End Dialogue System for Knowledge Grounded Conversation. arXiv preprint arXiv:170904264. 2017;. 